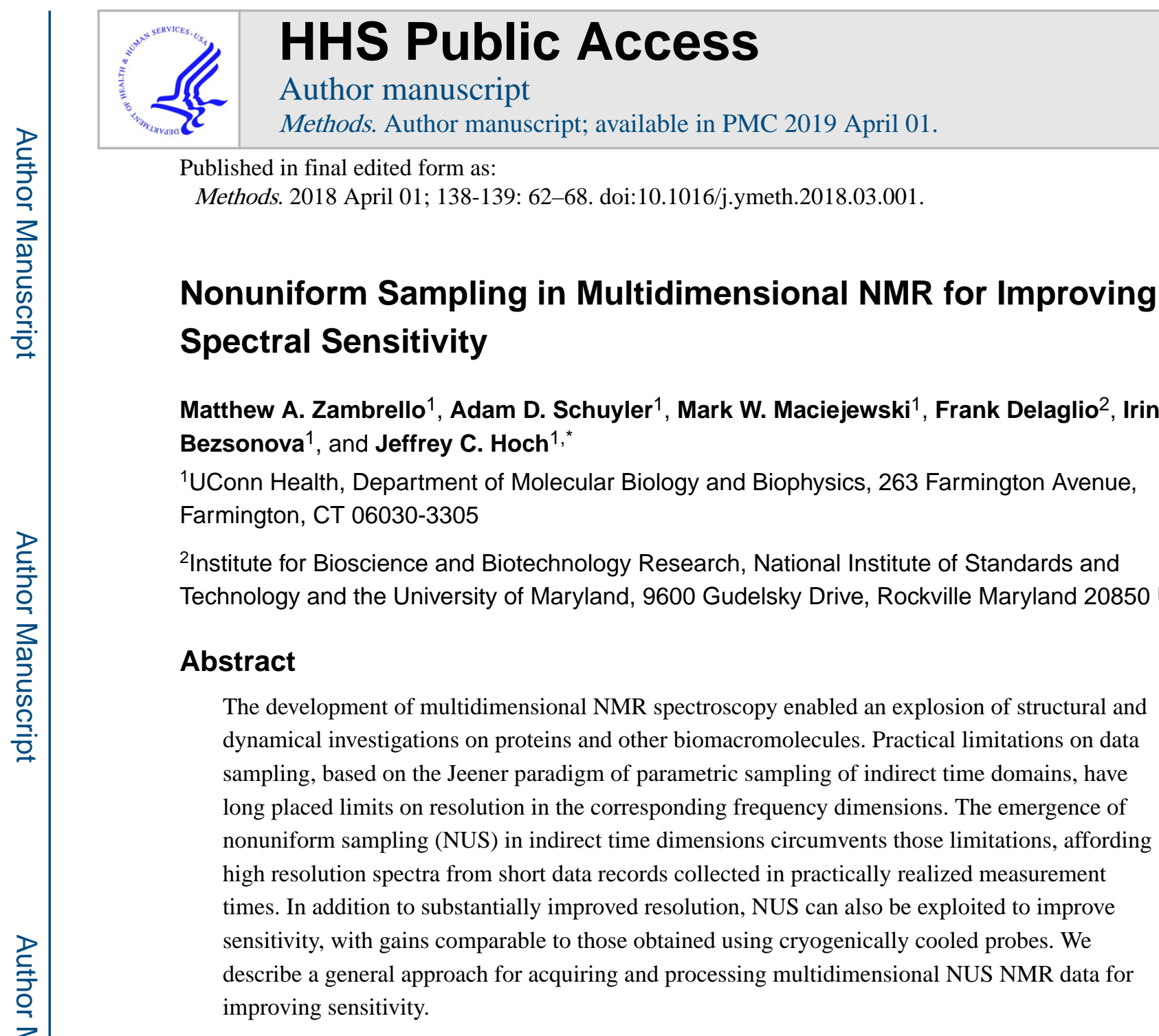

\title{
Introduction
}

The proposal by Jean Jeener ${ }^{1}$ to parametrically sample an indirect time domain by repeating pulsed NMR experiments with an incremented time delay lead to the development of modern multidimensional NMR experiments. This simple idea nevertheless had profound impact, because the application of NMR to complex biomolecules that is routine today would not be possible without the resolution afforded by multiple dimensions. Yet even early on, it was well appreciated that there were practical limits on the resolution achievable along indirect dimensions, as the discrete Fourier transform (DFT) requires long data records for high resolution. The first and still most widely-used method to ameliorate this sampling limitation is to use linear prediction ${ }^{2}$ (LP) to extrapolate the signal beyond the measured interval. As simple and powerful as this method is, it is often not robust, especially for noisy data ${ }^{3}$.

*Correspondence to hoch@uchc.edu.

Publisher's Disclaimer: This is a PDF file of an unedited manuscript that has been accepted for publication. As a service to our customers we are providing this early version of the manuscript. The manuscript will undergo copyediting, typesetting, and review of the resulting proof before it is published in its final citable form. Please note that during the production process errors may be discovered which could affect the content, and all legal disclaimers that apply to the journal pertain. 
LP extrapolation ${ }^{4}$ uses time domain data sampled at uniform intervals as input and numerically extends the data at the same uniform intervals, and is thus suitable for the DFT. A more general approach of sampling at nonuniform intervals would enable collection of data at long evolution times (needed for high resolution) while shortening data collection times. This requires some method other than the DFT for computing the spectrum of a time series collected at nonuniform intervals. Barna et al. ${ }^{5,6}$ used maximum entropy (MaxEnt) reconstruction, a method originally developed ${ }^{7}$ in radio astronomy, in the first demonstration of this general approach for multidimensional NMR. Appreciation of the power of nonuniform sampling (NUS) grew considerably with the introduction of radial sampling methods introduced by Kupce and Freeman ${ }^{8,9}$. Though their approach used back-projection to reconstruct the spectrum (and hence is often referred to as projection reconstruction), the ability of MaxEnt to processes radially-sampled data ${ }^{10}$ demonstrated that radial sampling is a special case of the general approach introduced by Barna and colleagues.

Over the past decade, research into optimal strategies for NUS and non-Fourier methods for spectral reconstruction from NUS data has accelerated. Methods based on $l_{1}$-norm minimization (whether via convex optimization or iterative fixed-point methods) are now commonly referred to as compressed sensing (CS) approaches ${ }^{11}$, and important theoretical work has led to theorems concerning the minimum number of samples needed to reliably recover a spectrum, and the universality of a sharp phase transition between success and failure ${ }^{12}$. Most of the theoretical work, however, is focused on random sampling (rarely used in NMR for reasons discussed below) and real-valued data (as opposed to the hypercomplex signals encountered in NMR). Thus while CS theorems have provided useful guidelines, they usually do not quantitatively apply to NMR, and thus the number of samples required for accurate recovery of multidimensional NMR spectra and the dependence on spectral sparsity need to be determined empirically.

Most reported applications of NUS and non-Fourier spectral reconstruction utilize NUS to reduce the measuring time required to obtain high resolution in the indirect dimensions ${ }^{13,14}$. It is well known (see especially the work of Rovnyak, Polenova, and Wagner ${ }^{15-21}$ ), however, that instead of saving measuring time, NUS can be used to improve the sensitivity per unit time for experiments for which the signal envelope is not constant in time. For an exponentially decaying sinusoid, sampling evolution times longer than $1.26 T_{2}$ results in diminishing sensitivity $3,18,22$. Conversely, resolving peaks separated by the natural linewidth typically requires sampling to an evolution time of $\pi T_{2}$. NUS affords a bridge between these seemingly incommensurate criteria: by sampling less frequently beyond evolution times of $1.26 T_{2}$ but reaching a maximum evolution time of $\pi T_{2}$, the sensitivity per unit measurement time can be dramatically increased over an experiment that acquires the same number of indirect dimension samples, sampled uniformly. Using NUS, the time saved for the samples not acquired is used to perform additional signal averaging for the samples that are acquired, which are predominantly less than $1.26 T_{2}$. For methods of spectrum analysis that are linear or nearly so, the sensitivity gain is given by the intrinsic signal-to-noise ratio (iSNR) determined in the time domain as defined by Rovnyak and colleagues ${ }^{15}$, modified to account for the number of transients signal-averaged for each tuple of indirect evolution times (il $S W(1), j / S W(2))$ 


$$
i S N R \propto \frac{\sum_{i=0}^{n_{1}-1} \sum_{j=0}^{n_{2}-1} K_{i j} e^{-i / S W(1) T_{2}(1)} e^{-j / S W(2) T_{2}(2)}}{\sqrt{\sum_{i=0}^{n_{1}-1} \sum_{j=0}^{n_{2}-1} K_{i j}}}
$$

where $T_{2}(1)$ and $T_{2}(2)$ are the time constants for the signal envelope decay in the indirect dimensions 1 and 2, $n_{1}$ and $n_{2}$ are the number of elements in the uniform sampling grid along dimensions 1 and 2, $\mathbf{K}$ is a sampling matrix with elements $K_{\mathrm{ij}}$ equal to zero for indirect time evolution tuples that are not sampled and equal to the number of FIDs accumulated for tuples that are sampled, and $S W(1)$ and $S W(2)$ are the spectral sweep widths in dimensions 1 and 2. iSNR simply captures the signal power in the measured signal relative to the noise power. Equation [1] is a general expression suitable for comparing the relative sensitivity of sampling schemes that have an arbitrary number of transients sampled for each tuple of indirect time evolutions, and is the discrete counterpart of the analytic expressions given by Rovnyak and colleagues. The use of proportionality rather than equality reflects the fact the there are many other factors that influence true sensitivity and are not reflected in the expression, however the proportionality permits comparison of sampling strategies when all other aspects of the experiment are held fixed. Note that Eq. (1) corresponds to two parametrically-sampled (indirect) time dimensions; it is easily generalized to arbitrary dimension. Nonuniform sampling is generally only useful in the indirect dimensions, i.e. 2D NUS is used for 3D experiments.

A useful way to bias the sampling to evolution times shorter than $1.26 T_{2}$ is to use exponential weighting of the sampling density that is related to the decay of the signal envelope. The term "matched sampling" refers to NUS in which the sampling density is

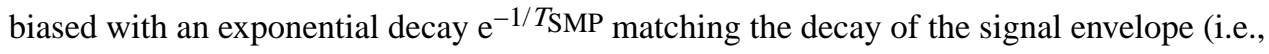
the decay time of the biased sampling time $T_{\mathrm{SMP}}$ is equal to $T_{2}$ ). In "overmatched sampling", a decay rate for the sampling density faster than that of the signal envelope is used (e.g. $2 \mathrm{X}$ overmatched sampling corresponds to $T_{\mathrm{SMP}}=T_{2} / 2$ ).

Figure 1 depicts $i S N R$ for exponentially biased sampling as a function of sampling coverage (the fraction of indirect time evolution tuples that are sampled from the uniform grid), assuming $40 \mathrm{~Hz}$ linewidths in dimensions 1 and 2 and a fixed number of samples $N=128 \times$ 128 or 16384, using sampling density decay constants $T_{\mathrm{SMP}}$ of either zero (uniform random), $T_{2}$, or $T_{2} / 2$ in both indirect dimensions. Values are reported relative to the iSNR for full uniform sampling, or sampling coverage of 1.0. The sampling bandwidth was chosen so that the maximum increment of 128 corresponds to a bit longer than $1.26 T_{2}$. "Time equivalent" iSNR is the iSNR obtained using the same number of FIDs as would be collected using uniform sampling of the $128 \times 128$ grid. A nonuniform sample schedule can be made to be time equivalent to a uniform sample schedule by increasing the number of transients for FIDs that are collected. This can be done by uniformly increasing the number of transients by roughly the inverse of the sample coverage (e.g. a 25\% coverage schedule that collects 4 transients per FID is time equivalent to uniform sampling with 1 transient per FID). Time equivalency for the NUS schedule can also be achieved by nonuniformly setting the number of transients by either following the sampling density of the sample schedule or 
by using any other density function. Results shown here are for single realizations of a sampling schedule generated according to each distribution function (and hence exhibit slight variation from expected statistical averages). The lower three curves in Figure 1 correspond to reduced overall measurement time, and the top three correspond to the same measuring time as full uniform sampling.

Time-equivalent uniform random sampling (green circles) has no impact on relative sensitivity, whereas exponentially biased sampling ( $T_{2}$ orange squares, $T_{2} / 2$ cyan triangles) result in improved sensitivity (approaching a factor of two for $T_{2} / 2$ biased sampling at $2 \%$ sampling coverage). This is consistent with the observations of Rovnyak et al. ${ }^{18}$, who demonstrated sensitivity gains exceeding a factor of two for sampling at low coverage and heavily biased to short times. All the curves approach the value 1 as coverage approaches 1 , where the sampling becomes uniform regardless of the sampling distribution employed.

As could be inferred from the independence of iSNR on sampling coverage for uniform random sampling, sensitivity gains that do accrue from biased sampling can only be realized when the signal envelope is not constant, for example modulated by exponential relaxation or J-modulation ${ }^{23}$. (In principle sampling can be tailored to match the J-modulation of the signal envelope, however sampling schedules designed in this way are very brittle, in the sense that slight deviations from the assumed J-modulation can drastically reduce their performance ${ }^{23}$.) In constant-time experiments the signal envelope is uniform, so there is no way to bias sampling toward times where the signal is stronger, and thus no way to improve the sensitivity per unit time; for these experiments, matched NUS is random sampling. For semiconstant-time experiments the signal envelope is an amalgam. In principle one could use random sampling for the initial constant-time period and exponential sampling for the real-time period, however in practice we find simply using exponentially biased sampling spanning the entire signal affords useful sensitivity gains.

The iSNR metric is a powerful and unbiased method for comparing the relative sensitivity of different sampling schemes, but it is not the entire story. iSNR considers only the amount of signal present in the empirical time domain data, and does not consider the method used to perform spectral analysis of the data. Only if the method used to compute the spectrum from the data has no impact on sensitivity are the inferences gleaned from iSNR complete. This is true for methods that are linear or nearly linear, and preserve norms (e.g. obey Parseval's Theorem ${ }^{4}$, which states that the integrated signal power is the same in the time and frequency domains). Many non-Fourier methods used to process NUS data are characteristically nonlinear. The nonlinearity of MaxEnt reconstruction is relatively well characterized, scaling down small signal values more than large signal values. A consequence of this nonlinearity is that apparent gains in signal-to-noise ratio do not necessarily correspond to gains in sensitivity ${ }^{24}$. It also implies that additional effort (beyond comparing iSNR values) is needed to fully characterize the relative sensitivity of NUS experiments that takes into account the method of spectrum analysis employed, if it is nonlinear.

We illustrate the sensitivity gains that can be achieved even when the signal envelope does not decay using data from an HNCACB experiment on the UBL3 domain from USP $7^{25}$. The 
experiment is semiconstant-time in the $t_{2}\left({ }^{15} \mathrm{~N}\right)$ dimension) and constant-time in the $t_{1}$ dimension $\left({ }^{13} \mathrm{C}\right)$, so there is little if any decay of the signal envelopes in $t_{1}$ and $t_{2}$ (interferograms depicting the signal envelopes in $t_{1}$ and $t_{2}$ of the US data are shown in Figure 2). Accordingly, iSNR (Eq. (1)) indicates that there is little sensitivity gain to be expected from NUS (iSNR for uniform sampling is 25.8, compared to 27.0 for the 25\% 2D sampling schedule depicted in Figure 3, using 4 times as many transients so the overall experiment time is the same). Figure 4 depicts projections of the HNCACB spectra onto the ${ }^{13} \mathrm{C}-{ }^{1} \mathrm{H}$ plane. Panel A (top) shows the DFT spectrum computed for uniformly sampled (US) data. Panel B shows the spectrum computed from the US data using MaxEnt reconstruction. Panel (C) is the MaxEnt spectrum computed for NUS data, collected using uniform random sampling in $t_{1}$ and $t_{2}$. However the results using MaxEnt are strikingly different for uniform sampling and NUS. The one-dimensional cross sections (taken at the position indicated by the dashed lines) suggest substantial improvement, and also appear to confirm that sensitivity gains from redeploying the time saved through NUS are not fully accounted for by iSNR alone.

To validate the apparent sensitivity gain, we performed in-situ Receiver Operating Characteristic (IROC) analysis ${ }^{26}$. In this recently-developed approach, synthetic timedomain signals are added to the empirical data, in relatively blank regions of the spectrum and having amplitudes that span the range from near the noise to the magnitude of the strongest empirical signals. Using these synthetic signals as ground truth, it is possible to compute true and false discovery rates (the number of true and false positives) as a function of detector (peak-picker) threshold. IROC analysis can be applied to arbitrary data processing workflows, and provides quantitative sensitivity and resolution metrics that enable comparison of linear or nonlinear methods of spectrum analysis. The IROC curve for the data shown in Fig. 2 is shown in Fig. 5. Panels A and B depict example cross-sections containing both empirical (near $7.8 \mathrm{ppm}{ }^{1} \mathrm{H}$ frequency) and synthetic peaks, using the US and NUS data sets. The signal amplitudes in panel B were multiplied by 4 to reflect the $4 \mathrm{X}$ signal averaging performed for the NUS data. In the 1D cross-sections corresponding to the vertical dashed lines, five synthetic peaks are discernible in the NUS spectrum (panel B), but only three are discernible in the US spectrum (panel A). Panel C shows the IROC curves for the spectra from panels A (cyan) and B (magenta). Values at the left correspond to high threshold values, where few peaks (whether true or false) are detected. As the detection threshold is lowered, more peaks are detected. For the majority of useful threshold values (where the curves are closest to the point $(0,1)$ corresponding to perfect detection, all real peaks detected and no false peaks detected), more true peaks are detected in the NUS data than in the US data.

\section{Example Workflow}

The basic steps involved in conducting and analyzing a NUS experiment consist of determining a suitable sampling schedule, performing the experiment, and reconstructing the spectrum. All tools, including the new version of NMRPipe used to implement the workflow described below are available on the NMRbox platform ${ }^{27}$ (http://NMRbox.org). 


\section{Generate schedule}

The Rowland NMR Toolkit includes a tool, sampsched2d for generating 2D sampling schedules that are random, exponentially biased, or sine-modulated in each dimension. Inputs are the total number of samples to be acquired, the filename for output, a seed for the pseudo-random number generator, and for each dimension the linewidth (in $\mathrm{Hz}$ ) corresponding to the sampling density decay rate, the J-modulation frequency (in $\mathrm{Hz}$; frequently zero), the maximum possible increment (integer), and the spectral width (in $\mathrm{Hz}$ ). The output is a text file consisting of two integers per line indicating the indices of the indirect time evolution in each dimension. The sampling schedule is used to perform the experiment and to reconstruct the spectrum. An additional tool implemented on the NMRbox platform, nus-tool, provides a graphical user interface and support for additional sampling schemes. Alternatively, the most recent software on commercial NMR instruments provide support for generating NUS schedules.

The details of optimal schedules remain an active area of research, but some useful rules-ofthumb have been derived from experience. The smallest useful sampling coverage depends on the sparsity of the spectrum, the dynamic range (difference between the weakest and strongest signal components), and the dimensionality of the experiment. For coherencetransfer experiments where the signals are expected to be similar in amplitude and the spectrum fairly sparse, we find that sampling coverage of around $30 \%$ per NUS dimension to be conservative, e.g $10 \%$ coverage for a 2D experiment. For NOESY experiments where the sparsity is lower (more signals are expected) and the dynamic range is high, we find that more samples are typically needed, around 50\% per NUS dimension. For dimensions where the signal envelope decays exponentially, we typically apply an exponential bias to the sampling distribution that decays twice as fast as the signal envelope. For constant-time or semiconstant-time dimensions, we typically use a uniform (random) distribution. Other sampling schemes have been proposed ${ }^{28,29}$, but none have yet been shown to be systematically superior.

\section{Reconstruct spectrum}

Many different non-Fourier methods have been utilized for computing spectra from NUS data ${ }^{30}$. Here we describe MaxEnt reconstruction as practiced in our laboratory ${ }^{4}$. ConstantAim and Constant $-\lambda$ : Processing data with Maximum Entropy Reconstruction (MaxEnt) as implemented in the Rowland NMR Toolkit (RNMRTK) can be performed in two modes, (1) constant-aim and (2) constant- $\lambda$. When processing all dimensions simultaneously with MaxEnt, including the acquisition dimension, constant-aim mode is used. When one or more of the dimensions are processed with the DFT, MaxEnt is performed in constant- $\lambda$ mode. ( $\lambda$ is the weighting factor applied to the constraint statistic that determines it's importance relative to the entropy; the value of $\lambda$ determines the extent of nonlinearities manifest in the MaxEnt spectrum.) In practice when processing data in constant $-\lambda$ mode a preliminary reconstruction in constant-aim mode is performed on a representative part of the spectrum to determine the converged $\lambda$ value. Processing the acquisition dimension with a DFT and processing all the indirect dimensions with MaxEnt is the general approach, since sampling is rarely limited in the acquisitions dimension and is assumed for the workflow described here. 
Adjustable parameters def and aim-MaxEnt requires two adjustable parameters; def and aim. Def represents the scale at which nonlinear effects become significant and aim is a measure of how tight the agreement between the reconstruction and the empirical data must be for convergence. Def values that are large will have nearly linear reconstructions, and if def is large and aim is small the results will be close to the DFT of a zero-filled spectrum. Very low values for $d e f$ will cause slow convergence and give rise to spiky noise distributions. Thus, determining reasonable values for def and aim are critical for obtaining quality reconstructions. In practice, reasonable reconstructions are obtained with a value for aim equal to or just above the noise power and a def value somewhat lower than the noise.

Automated determination of def, aim, and $\lambda-A n$ automated method for determining def, aim, and $\lambda$ has been implemented in the latest version of NMRPipe ${ }^{31}$ and starts with determining the noise power from the interferograms after the acquisition dimension is transformed with the DFT. It is critical that a region of the spectrum along the acquisition dimension be extracted to avoid issues from a strong solvent signal. From the noise power aim and def are set by the following equations and thus the only adjustable parameters become the def and aim scale factors with typical values of 0.2 (range $0.05-1$ ) and 1.0 (range $1-3$ ) respectively.

$$
\begin{gathered}
\text { aim }=(\text { Noise Power })(\text { Scale }) \quad[2 \mathrm{a}] \\
\text { def }=\left(\text { Noise Power } \frac{\sqrt{\text { Number of Complex FIDs collected }}}{\sqrt{\text { Product of Indirect Output Sizes }}}\right)(\text { Scale })
\end{gathered}
$$

During a MaxEnt reconstruction in constant-aim mode the final converged $\lambda$ value will be reported. In the automated workflow a MaxEnt reconstruction is performed in constant-aim mode with def and aim set as above and a log of the output captured and analyzed to average the ten highest $\lambda$ values. Reconstructions of noise will converge with smaller $\lambda$ values, thus taking the average of the highest $\lambda$ values gives a more reliable estimate. Another approach would be to only perform the constant-aim MaxEnt reconstruction on a representative part of the spectrum with stronger signals. Once a value is determined for $\lambda$ a final MaxEnt reconstruction is performed in constant $\lambda$ mode using $d e f$ as defined above and the averaged $\lambda$ value.

Processing Workflow in NMRPipe-A newer feature of NMRPipe is the ability to generate processing scripts from within NMRDraw and in the latest version installed in NMRbox, NUS data can be reconstructed with IST, SMILE, and MaxEnt as implemented in the RNMRTK. Below are the workflow steps.

- Data conversion and expansion - Convert data from vendor format to NMRPipe format and expand the NUS data with zeros for any points not collected using the "bruker" or "varian" programs of NMRPipe. 
- $\quad$ NMRDraw in process mode - The data is opened in NMRDraw in process mode with extranus parameters.

- $\quad n u D F T$ - With the data fully expanded a simple DFT of the full spectrum is performed allowing rapid determination of phase values, apodization, zero-fill sizes, ROI to extract, sign alterations, data reversals, and other parameters necessary for processing any spectrum. The $2 \mathrm{D}$ skyline projection views built into NMRPipe processing mode are helpful in parameter determination.

- $\quad$ Automated MaxEnt reconstruction - Reconstructing the spectrum with MaxEnt (RNMRTK) from within NMRPipe is fully automated. Internally scripts are generated to process the acquisition dimension with a DFT and the interferograms analyzed to determine the noise power used for automated determination of def and aim as described above. Data processed along the acquisition dimension is converted to RNMRTK format using Connjur Spectrum Translator $^{32}$. The converted data is reconstructed with MaxEnt (RNMRTK) in constant-aim mode using a default value of 1.0 for scaling aim and the output analyzed to average the ten highest $\lambda$ values. A second MaxEnt reconstruction is performed in constant $\lambda$ mode using the averaged $\lambda$ value from the constant-aim run. Finally, data is converted back to NMRPipe format and processing of the indirect dimensions performed for phasing, sign alterations, data reversals, deletion of the imaginary component, and creation of 2D projections for quick viewing of the spectrum. Note that a DFT in the indirect dimension is not performed as the output from MaxEnt (RNMRTK) is in the frequency domain.

\section{Concluding remarks}

\section{The challenge of quantifying sensitivity}

It has been known for decades that apparent improvements in SNR obtained using nonFourier methods do not necessarily correspond to improvements in sensitivity ${ }^{24}$, which is the ability to distinguish signal from noise. What we mean by "sensitivity" is very much determined by how we distinguish signals. If we employ linear processing methods (whether DFT or a non-Fourier method applied in a quasi-linear regime) and simply use the amplitude in the frequency domain to identify signals, then SNR is a reasonable proxy for sensitivity. However, any detection method that considers signal characteristics other than or in addition to amplitude will result in nonlinear detection. A simple example is a peak picker that considers lineshape and/or linewidth. Gains in sensitivity that can accrue from a nonlinearity are not captured by consideration of the amount of signal power captured by a sampling scheme (iSNR). Additional research in this area is needed.

The ability of NUS to achieve greater sensitivity per unit measuring time than uniform sampling when the signal envelope is not constant is well known, and although demonstrated previously by Rovnyak, Polenova, and Wagner and colleagues, it is not widely practiced. This is somewhat astonishing, because the gains that can be achieved are comparable to those from expensive cryogenically-cooled RF probes. NUS can be employed at zero cost, and software tools for implementing NUS experiments and analyzing the results are 
abundant (see NMRbox.org). Even though there is still much to learn about the sensitivity gains that accrue from NUS and from the non-Fourier methods used for spectral reconstruction from NUS data, it should become a standard technique for multidimensional NMR experiments.

\section{Acknowledgments}

We thank David Rovnyak for useful discussions. This work was supported by grants from the US National Institutes of Health (P41GM111135, R01GM123249). JCH is grateful to the Weizmann Institute for Science for a Joseph Meyerhoff Visiting Faculty Fellowship and to the Frydman group for their hospitality during completion of this manuscript.

\section{References}

1. Jeener, J. Oral Presentation. Ampere International Summer School, Basko Polje Yugoslavia; 1971.

2. Olejniczak ET, Eaton HL. Extrapolation of time-domain data with linear prediction increases resolution and sensitivity. J Magn Reson. 1990; 87:628-632.

3. Stern AS, Li KB, Hoch JC. Modern spectrum analysis in multidimensional NMR spectroscopy: comparison of linear-prediction extrapolation and maximum-entropy reconstruction. J Am Chem Soc. 2002; 124:1982-1993. [PubMed: 11866612]

4. Hoch, JC., Stern, AS. NMR Data Processing. Wiley-Liss; 1996.

5. Barna JCJ, Laue ED. Conventional and exponential sampling for 2D NMR experiments with application to a 2D NMR spectrum of a protein. J Magn Reson. 1987; 75:384-389.

6. Barna JCJ, Laue ED, Mayger MR, Skilling J, Worrall SJP. Exponential sampling, an alternative method for sampling in two-dimensional NMR experiments. Journal of Magnetic Resonance (1969). 1987; 73:69-77.

7. Skilling J, Bryan R. Maximum entropy image reconstruction: general algorithm. Mon Not R Astron Soc. 1984; 211:111-124.

8. Freeman R, Kupce E. Distant Echoes of the Accordion: Reduced Dimensionality, GFT-NMR and Projection-Reconstruction of Multidimensional Spectra. Concepts in Magn Reson. 2004; 23:63-75.

9. Kupče E, Freeman R. Projection-reconstruction technique for speeding up multidimensional NMR spectroscopy. J Amer Chem Soc. 2004; 126:6429-6440. [PubMed: 15149240]

10. Mobli M, Stern AS, Hoch JC. Spectral reconstruction methods in fast NMR: reduced dimensionality, random sampling and maximum entropy. J Magn Reson. 2006; 182:96-105. DOI: 10.1016/j.jmr.2006.06.007 [PubMed: 16815055]

11. Donoho DL. Compressed Sensing. IEEE Transactions on Information Theory. 2006; 52:12891306.

12. Monajemi, H. PhD thesis. Stanford University; 2016. Phase Transitions in Deterministic Compressed Sensing, with Application to Magnetic Resonance Spectroscopy.

13. Billeter, M., Orekhov, V. Topics in Current Chemistry. Springer; 2012.

14. Mobli, M., Hoch, JC. Royal Society of Chemistry; London: 2017.

15. Palmer MR, et al. Sensitivity of Nonuniform Sampling NMR. J Phys Chem B. 2015; 119:65026515. [PubMed: 25901905]

16. Palmer MR, Wenrich BR, Stahlfeld P, Rovnyak D. Performance tuning nonuniform sampling for sensitivity enhancement of signal-limited biological NMR. J Biomol NMR. 2014; 58:303-314. DOI: 10.1007/s10858-014-9823-5 [PubMed: 24682944]

17. Paramasivam S, et al. Enhanced Sensitivity by Nonuniform Sampling Enables Multidimensional MAS NMR Spectroscopy of Protein Assemblies. Journal of Physical Chemistry B. 2012; 116:7416-7427. DOI: 10.1021/Jp3032786

18. Rovnyak D, Sarcone M, Jiang Z. Sensitivity enhancement for maximally resolved two-dimensional NMR by nonuniform sampling. Magn Reson Chem. 2011; 49:483-491. [PubMed: 21751244]

19. Rovnyak D, Hoch JC, Stern AS, Wagner G. Resolution and sensitivity of high field nuclear magnetic resonance spectroscopy. J Biomol NMR. 2004; 30:1-10. [PubMed: 15452430] 
20. Hyberts SG, Robson SA, Wagner G. Interpolating and extrapolating with hmsIST: seeking a tmax for optimal sensitivity, resolution and frequency accuracy. J Biomol NMR. 2017; 68:139-154. DOI: 10.1007/s10858-017-0103-z [PubMed: 28332026]

21. Hyberts SG, Robson SA, Wagner G. Exploring signal-to-noise ratio and sensitivity in nonuniformly sampled multi-dimensional NMR spectra. J Biomol NMR. 2013; 55:167-178. DOI: 10.1007/s10858-012-9698-2 [PubMed: 23274692]

22. Matson GB. Signal integration and the signal-to-noise ratio in pulsed NMR relaxation measurements. J Magn Reson. 1977; 25:477-480.

23. Schmieder P, Stern AS, Wagner G, Hoch JC. Application of nonlinear sampling schemes to COSYtype spectra. J Biomol NMR. 1993; 3:569-576. [PubMed: 8219741]

24. Donoho DL, Johnstone IM, Stern AS, Hoch JC. Does the maximum entropy method improve sensitivity? Proc Natl Acad Sci U S A. 1990; 87:5066-5068. [PubMed: 11607089]

25. Pozhidaeva AK, et al. Structural Characterization of Interaction between Human Ubiquitin-specific Protease 7 and Immediate-Early Protein ICP0 of Herpes Simplex Virus-1. J Biol Chem. 2015; 290:22907-22918. DOI: 10.1074/jbc.M115.664805 [PubMed: 26224631]

26. Zambrello MA, Maciejewski MW, Schuyler AD, Weatherby G, Hoch JC. Robust and transferable quantification of NMR spectral quality using IROC analysis. J Magn Reson. 2017; 285:37-46. DOI: 10.1016/j.jmr.2017.10.005 [PubMed: 29102819]

27. Maciejewski MW, et al. NMRbox: A Resource for Biomolecular NMR Computation. Biophys J. 2017; 112:1529-1534. DOI: 10.1016/j.bpj.2017.03.011 [PubMed: 28445744]

28. Worley B, Powers R. Deterministic multidimensional nonuniform gap sampling. J Magn Reson. 2015; 261:19-26. DOI: 10.1016/j.jmr.2015.09.016 [PubMed: 26524650]

29. Hyberts SG, Milbradt AG, Wagner AB, Arthanari H, Wagner G. Application of iterative soft thresholding for fast reconstruction of NMR data non-uniformly sampled with multidimensional Poisson Gap scheduling. J Biomol NMR. 2012; 52:315-327. DOI: 10.1007/s10858-012-9611-z [PubMed: 22331404]

30. Mobli M, Hoch JC. Nonuniform sampling and non-Fourier signal processing methods in multidimensional NMR. Prog Nucl Magn Reson Spectrosc. 2014; 83C:21-41. DOI: 10.1016/ j.pnmrs.2014.09.002

31. Delaglio F, et al. NMRPipe: a multidimensional spectral processing system based on UNIX pipes. J Biomol NMR. 1995; 6:277-293. [PubMed: 8520220]

32. Nowling RJ, et al. CONNJUR spectrum translator: an open source application for reformatting NMR spectral data. J Biomol NMR. 2011; 50:83-89. [PubMed: 21409563] 
Nonuniform sampling of indirect time dimensions is widely used in multidimensional NMR spectroscopy to shorten experiment times and improve resolution. Careful design of nonuniform sampling schemes can also be used to improve sensitivity. A protocol for obtaining sensitivity gains comparable to those attained with cryogenically-cooled probes is described. 


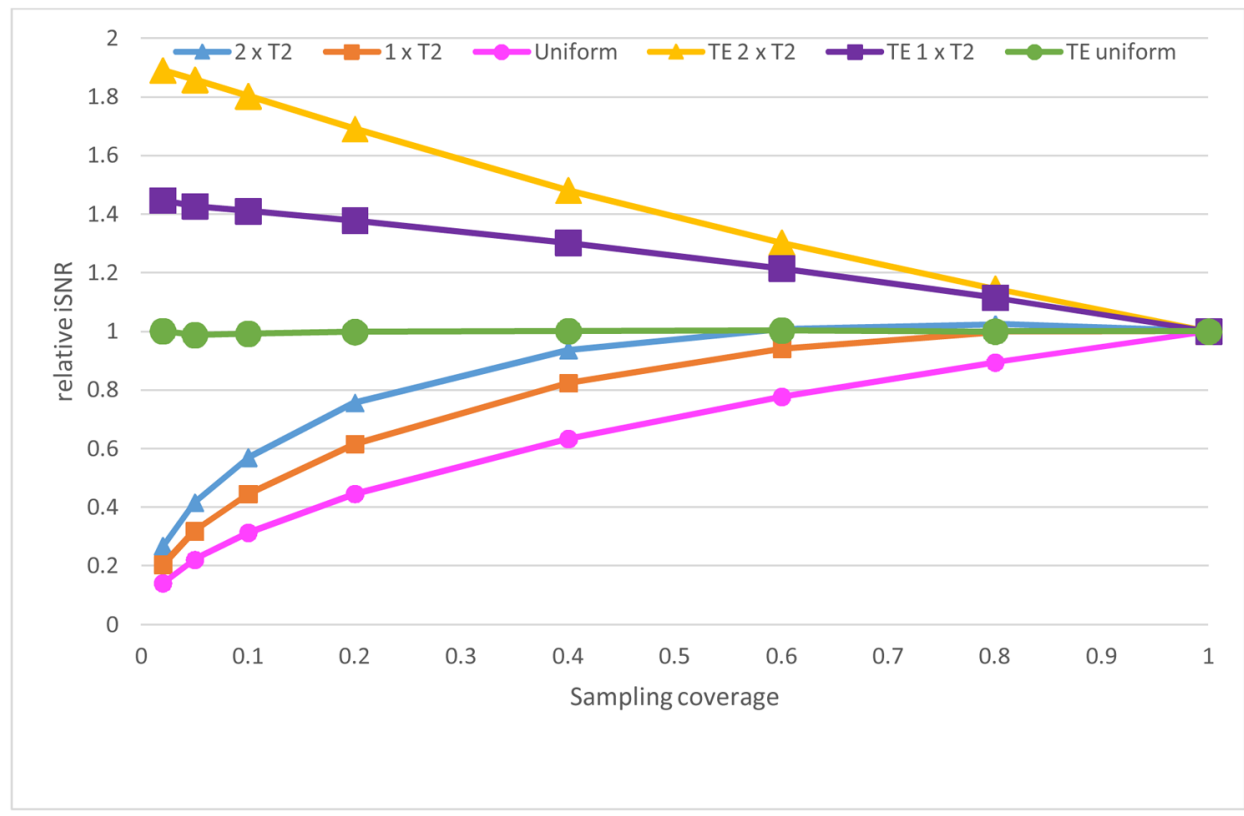

Figure 1.

iSNR as a function of coverage and decay rate for a 2D schedule, for different sampling decay rates ( $1 \mathrm{X}$ and $2 \mathrm{X}$ the signal envelope decay rate). iSNR decreases with decreasing coverage. However, when the number of transients is increased so that the overall experiment time is held constant, iSNR improves with decreasing coverage. Note the timeequivalent iSNR for random (unweighted) sampling is independent of sampling coverage. 

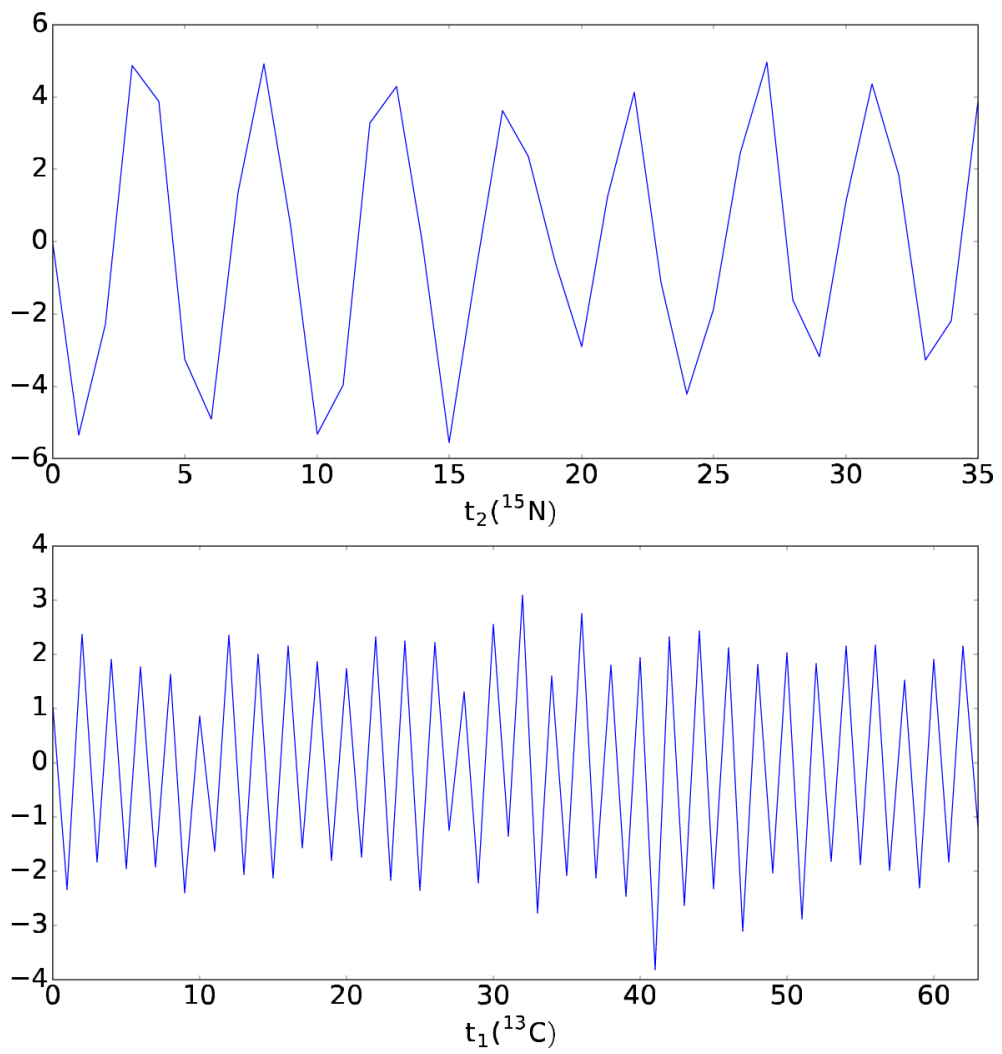

Figure 2.

Interferograms along $t_{1}$ and $t_{2}$ following DFT of the ${ }^{1} \mathrm{H}\left(t_{3}\right)$ dimension of the uniformly sampled (US) HNCACB data for the UBL3 domain of USP7. 


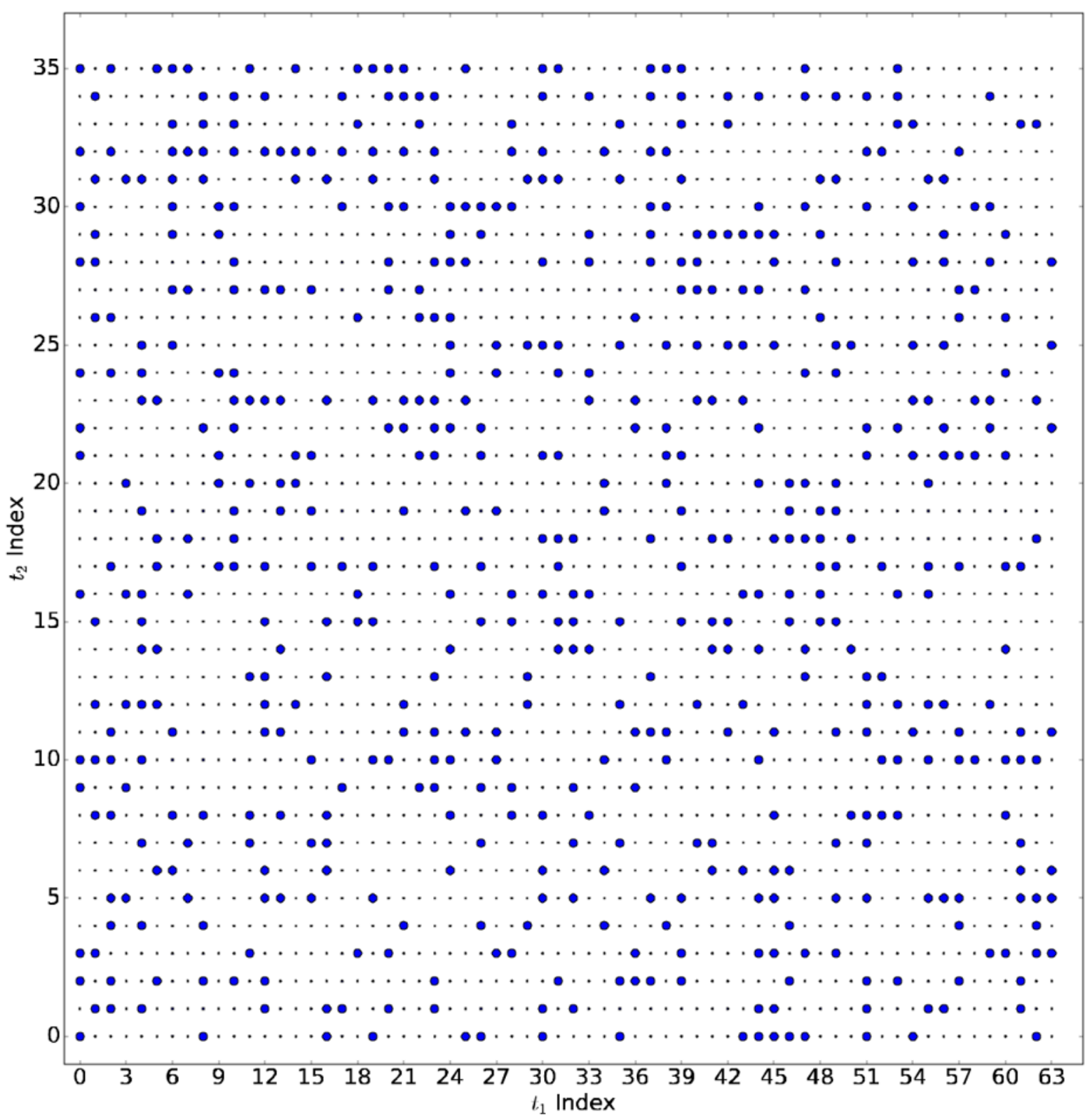

Figure 3.

The sampling schedule used to conduct the NUS HNCACB experiment for the UBL3 domain of USP7. Large dots indicate tuples of evolution times collected, small dots correspond to tuples not collected. The sampling coverage of $25 \%$ enabled $4 \mathrm{x}$ signal averaging at each tuple of evolution times, compared to uniform sampling, while maintaining the same overall measuring time. iSNR for the sampling schedule is 27.0, compared to 25.8 for uniform sampling. 


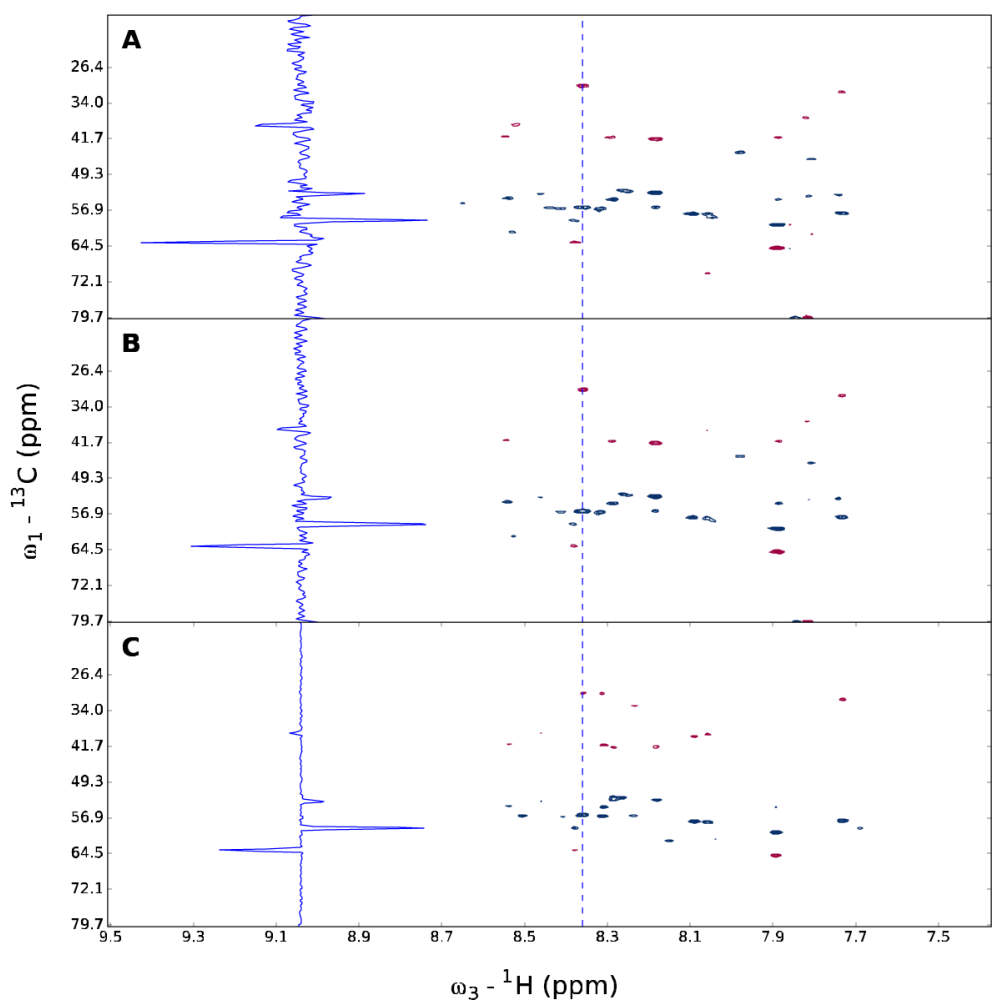

Figure 4.

Projections onto the ${ }^{13} \mathrm{C}-{ }^{1} \mathrm{H}$ plane and 1D cross-sections of HNCACB spectra for UBL3 from USP7. A. DFT spectrum obtained from US data. B. MaxEnt reconstruction from the US data. C. MaxEnt reconstruction from NUS data, 25\% coverage. The sampling schedule is depicted in Figure 3. The 1D cross-sections parallel to the ${ }^{13} \mathrm{C}$ axis are taken at the ${ }^{1} \mathrm{H}$ frequency indicated by the dashed line. 

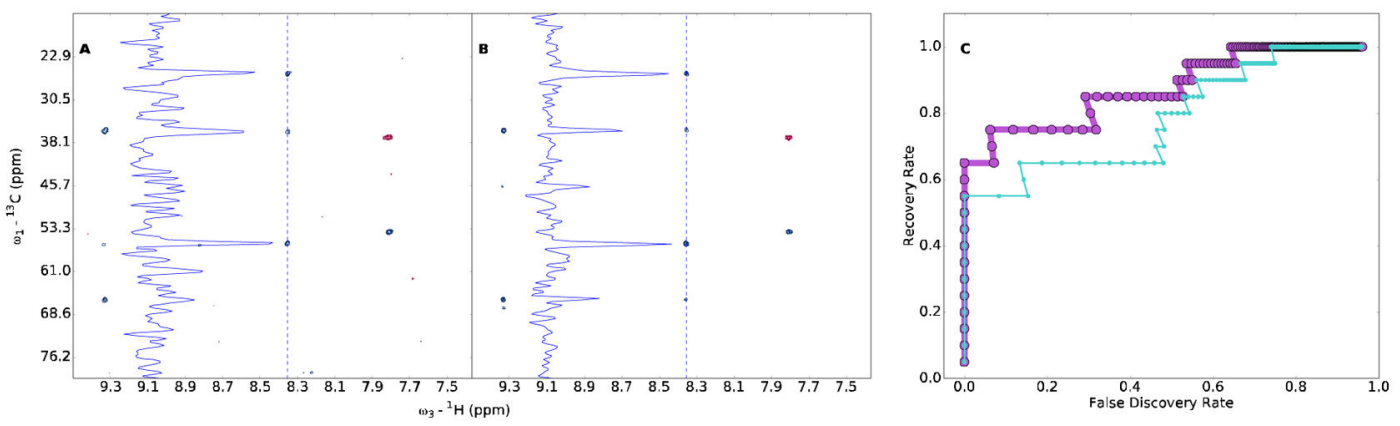

Figure 5.

IROC analysis performed for the data used in Fig. 4. Panels A and B depict a 2D crosssection containing both empirical (near $7.8 \mathrm{ppm} 1 \mathrm{H}$ ) and synthetic peaks. Panel A is derived from the US data set, Panel B from the NUS data set. The amplitudes of the syntactic signals added to the NUS data set are multiplied by 4 relative to the amplitudes of the signals added to the US data set, to reflect the $4 \mathrm{X}$ signal averaging in the NUS data. Panel $\mathrm{C}$ shows the IROC curves (US in cyan, NUS in magenta). 